\title{
Mass Spectrometry OMICS Approach to Study Medicinal Chemical Molecular Responses on Living Organisms
}

\section{Eduardo C Meurer*}

Fenn Laboratory of Mass Spectrometry, Exact Sciences Center, Federal University of Paraná, Paraná, Brazil

Studies on the effect of drugs on organisms such as plants, animals and human beings have experienced an evolutionary leap in the form of proteomic and metabolomic analysis using mass spectrometry (MS). The 2002, Chemistry Nobel prize winner John Fenn established a landmark in this field by introducing the electrospray ionization technique (ESI), which allows for the transfer of proteins and small biomolecules from solutions to mass spe 'ctrometers for MS analysis $[1,2]$. Since the advent of ESI-MS, a great variety of methodologies have appeared to help scientists in solving important questions on clinical and medicinal chemistry. MS has been now established as the stateof-the-art analytical tool in which richest data from molecular systems can be acquired. In medicinal chemistry, three MS approaches might be highlighted as the most prominent: data dependent UPLC-ESIHRMS metabolomics [3], data dependent nanoLC-nanoESI-HRMS proteomics [4] and MS imaging applied to the biomarker screening of tissues [5].

Metabolomic MS screening can usually be categorized into untargeted [6] and targeted analysis [7]. In untargeted metabolomics, as many molecules as possible are identified in a sample. Relative quantities of analytes can be determined when comparing treatments. Targeted metabolomics focuses on the relative or absolute quantitation of molecular targets using internal standards and/or analytical curves. Some MS approaches in metabolomic analysis have been interested in the identification of metabolites that are formed in vivo out of administered medication. The metabolism is directly related with both toxic effects and activity. Another field on interest in metabolomics is the study of how the regular metabolism of organisms is influenced by an administered medication.

The metabolic approach uses both high volume data obtained from chromatographic separation, as well as high-resolution MS data. These accurate data provide information of molecular formulas with errors around $1 \mathrm{ppm}$. Due to the high velocity of MS acquisition, tandem MS data from target constituents can be used to increase identification accuracy of thousands of small molecules in just one analytical run. This MS/MS data facilitates in the formation of an overview of present molecules per sample. Data handling using PCA or PLS statistical approaches is used to relate treatment evaluation with metabolite up and down regulation. For selectivity improvement in metabolomics, MS coupled to ion-mobility mass spectrometry can be used, in which molecules are separated according to their shape and then characterized even more reliably allowing the discrimination between isomeric or isobaric metabolites.

Proteomics analysis is currently conducted using nanoLCnanoESI-HRMS. It is therefore expected that this technique will soon be applied to the analysis of living organisms. The correlation of the proteome with metabolomics could be seen as the Holy Grail of how living organisms' work by being able to gather information that combines genomics-proteomics-metabolomics all together. Such as in metabolomics, proteomics is performed either in an untargeted [4] or targeted fashion [8]. Untargeted proteomic might find expressed proteins triggered by the medication administered to living organisms. For that it uses the speciation approach and gives clues about how the medicines interact towards upregulated and downregulated protein responses related to different treatments. Targeted Proteomics is aimed to reveal the role of specific proteins and for that uses selected reaction monitoring to quantify them relatively or absolutely using standards of proteotypic peptides and isotopically labeled peptides. For selectivity improvement in proteomics, MS coupled to ion-mobility is a powerful approach [9], in which peptides are separated according to their shape and then characterized even more reliably allowing the discrimination between isomeric or isobaric molecules. Another new approach is the data independent analysis that uses broad selection window to fragment peptides without any previous conditions usually adjusted to data-dependent analysis [10]. One class of interesting proteins that can be targeted via MS proteomics is the cytokines. The proteomic approach may lead to clues on how to improve the medication to act more effectively on a specific organism to which it is administered.

MS imaging uses a variety of MS techniques to inspect the chemical composition of a surface. Without the need for laborious sample preparation, ambient ionization techniques are rapidly providing highly valuable results in IMS [11]. One of such techniques, DESI, usually is coupled to high-resolution mass spectrometers or tandem MS systems to improve reliability in identification and sensitivity. The observed results are usually related to lipid distribution (lipidomics) of the analyzed tissue. Spectra from tissues reveal preferentially the best ionizable components such as phospholipids, diacylglycerols, triacylglycerols and free carboxylic acids, otherwise imaging drugs and metabolites in biological tissues were shown to be possible [12]. IMS has been causing a great impact on clinical and medical procedures for instance by helping pathologists to make better decisions on tumor interpretation or delineation after or during brain surgery. DESI-MS has been used successfully, for instance, to screen for cancer biomarkers and their $2 \mathrm{D}$ and $3 \mathrm{D}$ spatial distribution on a tissue.

The use of MS techniques on medicinal chemistry is highly valuable due to the high-level of spatial information at the molecular level associated with the speed and simplicity of analysis and the ability to answer important questions about how organic molecules might interact positively or negatively on living organism and its pathologies. The fast developments of new state-of-the-art MS techniques are providing increasingly higher sensitivity, speed and simplicity and higher amounts of detailed molecular information in both proteomics and metabolomics. There is, however, lots of improvements and discoveries to be made and a rough route lies ahead of us to collect and incorrectly interpret the huge amount of information from the most complex and sophisticated, and exquisitely designed thing on earth: Life.

*Corresponding author: Eduardo C. Meurer, Fenn Laboratory of Mass Spectrometry, Exact Sciences Center, Federal University of Parana, Jandaia do Sul, Parana, Brazil, Tel: +55(41) 3208-6192; E-mail: eduardo.meurer@ufpr.br

Received October 21, 2015; Accepted November 02, 2015; Published November 06,2015

Citation: Meurer EC (2015) Mass Spectrometry OMICS Approach to Study Medicinal Chemical Molecular Responses on Living Organisms. Med chem 5: 470471. doi:10.4172/2161-0444.1000303

Copyright: (c) 2015 Meurer EC. This is an open-access article distributed under the terms of the Creative Commons Attribution License, which permits unrestricted use, distribution, and reproduction in any medium, provided the original author and source are credited. 
Citation: Meurer EC (2015) Mass Spectrometry OMICS Approach to Study Medicinal Chemical Molecular Responses on Living Organisms. Med chem 5: 470-471. doi:10.4172/2161-0444.1000303

\section{References}

1. Yamashita M, Fenn JB (1984) Electrospray ion source. Another variation on the free-jet theme. J Chem Phys 80: 445.

2. Fenn JB, Mann M, Meng CK, Wong SF, Whitehouse CM (1989) Electrospray ionization for mass spectrometry of large biomolecules. Science 246: 64-71.

3. Dettmer K, Aronov PA, Hammock BD (2007) Mass spectrometry-based metabolomics. Mass Spectrom Rev 26: 51-78.

4. Aebersold R, Mann M (2003) Mass spectrometry-based proteomics. Nature 422: 198-207.

5. Takats Z, Wiseman JM, Gologan B, Cooks RG (2004) Mass spectrometry sampling under ambient conditions with desorption electrospray ionization. Science 306: 471-473

6. Evans AM, DeHaven CD, Barrett T, Mitchell M, Milgram E (2009) Integrated, Nontargeted Ultrahigh Performance Liquid Chromatography/Electrospray Ionization Tandem Mass Spectrometry Platform for the Identification and Relative Quantification of the Small-Molecule Complement of Biological Systems. Anal Chem 81: 6656-6667.
7. Altmaier E, Ramsay SL, Graber A, Mewes HW, Weinberger KM, et al. (2008) Bioinformatics analysis of targeted metabolomics-uncovering old and new tales of diabetic mice under medication. Endocrinology 149: 3478-3489.

8. Ong SE, Mann M (2005) Mass spectrometry-based proteomics turns quantitative. Nat Chem Biol 1: 252-262.

9. Valentine SJ, Plasencia MD, Liu X, Krishnan M, Naylor S, et al. (2006) Toward plasma proteome profiling with ion mobility-mass spectrometry. J Proteome Res 5: 2977-2984.

10. Tsou CC, Avtonomov D, Larsen B, Tucholska M, Choi H, et al. (2015) DIA Umpire: comprehensive computational framework for data-independent acquisition proteomics. Nat Methods 12: 258-264.

11. Wu C, Dill AL, Eberlin LS, Cooks RG, Ifa DR (2013) Mass spectrometry imaging under ambient conditions. Mass Spectrom Rev 32: 218-243.

12. Wiseman JM, Ifa DR, Zhu Y, Kissinger CB, Manicke NE, et al. (2008) Desorption electrospray ionization mass spectrometry: Imaging drugs and metabolites in tissues. Proc Natl Acad Sci USA 105: 18120-18125. 